\title{
A new genus and species of Ephydridae (Diptera) from the Oriental Region
}

\author{
JUNHUA ZHANG, DING YANG \& WAYNE N. MATHIS \\ (JZ \& DY) Department of Entomology, China Agricultural University, Beijing 100094, China. (WNM) Depart- \\ ment of Entomology, Smithsonian Institution, NHP 169, PO Box 37012, Washington, DC 20013-7012, USA
}

\begin{abstract}
A new genus and species of the tribe Dagini (subfamily Ephydrinae), Sinops sichuanensis, are described from specimens collected in China (Sichuan: Emeishan Mountain), and three species formerly comprising "the fluvialis group" of Psilephydra Hendel are transferred to the new genus ( $S$. fluvialis (Miyagi), S. kaskiensis (Mathis) and S. nepalensis (Mathis)) as new combinations. A cladistic analysis of the new genus with related genera in the tribe Dagini is presented and discussed, and keys to the genera of Dagini and to the species of the new genus are presented.
\end{abstract}

Key words: Diptera, Ephydridae, Sinops, new genus, new species, China

\section{Introduction}

We recently studied material collected in the Sichuan Province of China and discovered an undescribed species that belongs to a clade that had been recognized as "the fluvialis group" of Psilephydra Hendel (Mathis \& Zatwarnicki 1988). This group of four species, including a new species described below, is sufficiently distinct morphologically from Psilephydra that we are here describing it as a new genus in the tribe Dagini, subfamily E phydrinae. In addition to its morphological distinctiveness, and as a further indication of its generic status, our preliminary phylogenetic assessment of the group positions it as the sister group to the lineage giving rise to Physemops Cresson and Psilephydra (Fig. 16). Although describing the genus and species is the primary objective of this paper, we also provide a phylogenetic context for these descriptions as a cladistic analysis of the genus and related genera. To facilitate identification of the genus and its included species, we are also providing a revised key to the genera of Dagini and a key to the species of the new genus. 
The subfamily Ephydrinae is the $2^{\text {nd }}$ largest in the shore-fly family Ephydridae with 29 genera and 460 species (Mathis \& Zatwarnicki 1995). Of the four tribes included in Ephydrinae (Ephydrini, Dagini, Parydrini, Scatellini), the tribe Dagini is the most recently proposed in the nomenclatural history of the subfamily (Mathis 1982) and it also has the fewest number of genera and species (number of species indicated in parenthesis): Brachydeutera Loew (15 spp.), Dagus Cresson (8 spp.), Diedrops Mathis \& Wirth (4 spp.), Physemops Cresson (6 spp.) and Psilephydra Hendel (6 spp.). With the exception of Brachydeutera, which seems to be an anomaly and outlier in Dagini, the known distribution of the included taxa is the New World tropics and subtropical and tropical Asia. As we have just alluded, we consider the placement of Brachydeutera in Dagini to be provisional, awaiting more specific elaboration and documentation by syapomorphies. Biologically, with the exception of Brachydeutera, the species of Dagini are associated with fast flowing, well-oxygenated streams, including water falls and cascades (Mathis \& Zatwarnicki 1988, Mathis \& Hogue 1986).

\section{Material and Methods}

The descriptive terminology, with the exceptions noted in Mathis (1986), and Mathis and Zatwarnicki (1990a), and below, follows that published in the Manual of Nearctic Diptera (McAlpine 1981). We have followed the terminology for most structures of the male genitalia that other workers in Ephydridae have used (see references in Mathis 1986, Mathis and Zatwarnicki 1990a, 1990b), such as surstylus. The following abbreviations are used: acr - acrostichal, $a d$ - anterodorsal, $a v-$ anteroventral, $d c$ - dorsocentral, $n p l-$ notopleural, oc - ocellar, orb orbital, presc - prescutellar, $p s a$ - postalar, $p v$ - posteroventral, $s a$ supra-alar, $s c$ scutellar. The species description is composite and not based solely on the holotype.

The holotype and most paratypes are deposited in the Entomological Museum of China Agricultural University, Beijing; two paratypes are also deposited in the National Museum of Natural History (USNM), Washington, D.C.

The phylogenetic analysis, including successive weighting, was performed with the assistance of Hennig868 (Version 1.5; Farris 1988), a computerized algorithm that produces cladograms by parsimony analysis. Each morphological character was numerically coded and entered into a matrix, and the character data were polarized using outgroup procedures and for which the following codes were used: $0=$ plesiomorphic state, $1-2=$ apomorphic state, ? = state inapplicable. The taxon-character matrix is given in Table 1 . Although autapomorphies were not included in the cladistic analysis (they were made inactive), which would skew the consistency and retention indices, we have listed them on the cladogram and have included them as part of generic treatments and phylogenetic considerations to document the monophyly of lineages, particularly at the generic level. 
Dagini Mathis, 1982: 3. Type genus: Dagus Cresson, 1935. Mathis and Zatwarnicki 1988: 106-121 [revision], 1995: 229-235 [world catalog].

\section{Key to the Genera and Species Groups of Dagini Mathis}

1. Costal vein short, extended only to vein $\mathrm{R}_{4+5}$; face with distinct, mediovertical carina, extended from ptilinal suture to oral margin; arista distinctly pectinate, longest rays subequal to length of flagellomere 1 ........... Brachydeutera Loew

- Costal vein long, extended to vein M; face evenly transversely arched, not carinate; arista essentially bare to subpectinate with longest rays not more than $1 / 2$ length of flagellomere 1.

2. Pulvilli lacking; postpronotum with 1 to a few setulae............................Dagus Cresson

- Pulvilli present, conspicuous; postpronotum bare. 3

3. Distance between apices of veins $\mathrm{R}_{2+3}$ and $\mathrm{R}_{4+5}$ short, less than half distance between veins $\mathrm{R}_{4+5}$ and $\mathrm{M}$; gena high, equal to or greater than eye height; genal seta well developed and conspicuous; prescutellar acr setae well developed; propleuron setulose; 5th tarsomere with dorsoapical process extended beyond base of tarsal claws

Diedrops Mathis and Wirth

- Distance between apices of veins $R_{2+3}$ and $R_{4+5}$ subequal to that between veins $R_{4+5}$ and $\mathrm{M}$; gena short, usually not more than $1 / 2$ eye height; genal seta, if present, weakly developed and inconspicuous; prescutellar acr setae not evident; propleuron without setulae; 5th tarsomere not as above...

4. Two to 3 large, postsutural dc setae; arista mostly bare, at most with small rays (their lengths less than aristal width at base) along basal 1/4

- One large, postsutural dc seta inserted near scutellum; arista pectinate or macropubescent along at least basal 2/3 (genus Physemops Cresson)...6

5. Anterior npl seta weakly developed, much smaller than the posterior seta; forefemur with posteroventral row of short, spinelike setae. Psilephydra Hendel

- Anterior npl seta well developed, subequal in length to posterior seta; forefemur unarmed, lacking spinelike setae. Sinops, gen. nov.

6. Knob of halter black; ocellar bristles lacking; arista long, over twice combined length of first 3 antennal segments; vein $\mathrm{CuA}_{1}$ along posterior margin of discal cell bowed posteriorly the nemorosus group

- Knob of halter pale, usually yellowish; ocellar setae present, conspicuous; arista shorter, rarely over twice combined length of first 3 antennal segments; vein $\mathrm{CuA}_{1}$ along posterior margin of discal cell straight. the panops group 
Type species. Sinops sichuanensis sp. nov.

Diagnosis. Male body length 2.2-2.4 mm, wing length 2.5-2.7 mm. Head black, subshiny; frons dark colored, usually contrasted with lighter facial coloration. Two pairs of strong lateroclinate orb; medial facial area and lower facial margin with setae, of which 3-4 setae are lengthened laterally; oral setae well developed; gena with 1 distinct seta longer and stronger than other genal setae. Arista with short pubescent-like hairs.

Thorax generally black, subshiny. Three pairs of postsutural dc, presutural dc absent (except in S. nepalensis); acr weak and short, continuing in a posts row to base of scutellum; 1 strong presutural seta; posterior npl much farther from notopleural suture than anterior npl; katepisternal seta weaker than anepisternal seta; 1 strong sa, psa absent; 2 pairs of sc. Legs subshiny black. Forefemur with row of pv, and without row of spine-like setae, midfemur with row of av. Pulvilli well developed; claws short and distinctly curved. Wing: $\mathrm{C}$ extended to apex of $\mathrm{M}$; distance between $\mathrm{R}_{2+3}$ and $\mathrm{R}_{4+5}$ less than that between $\mathrm{R}_{4+5}$ and $\mathrm{M}$ but more than $1 / 2$ that between $\mathrm{R}_{4+5}$ and $\mathrm{M}$.

Abdomen generally black, subshiny. Male genitalia: Epandrium large and broad, parallel-sided in posterior view; cerci long, thickened at base and slender at apex; gonite falcate at base in lateral view. Female genitalia: Cerci rather thick, nearly trapezoidal in lateral view, brownish yellow at base and yellow at apex. Sternite 8 divided into 2 nearly triangular sclerites.

Distribution. The genus is known only from Asia and is currently represented by four species.

Etymology. Sinops is a Latinized combination of sino, meaning of or pertaining to China and the Chinese, and ops, meaning eye or face and which is a common generic ending in the tribe Dagini.

Remarks. Sinops is in the tribe Dagini and comprises what was formerly known as "the fluvialis group" of Psilephydra (Mathis and Zatwarnicki 1988), and the species of that group are here transferred to Sinops as new combinations: S. fluvialis (Miyagi 1977; Japan), S. kaskiensis (Mathis 1988; Nepal) and S. nepalensis (Mathis 1988; Nepal).

Sinops and Psilephydra are similar in having three postsutural dc. Sinops is distinguished from Psilephydra and other genera of the tribe Dagini by the well-developed lateral facial setae; the well-developed anterior notopleural seta; the distance between $R_{2+3}$ and $R_{4+5}$ being less than that between $R_{4+5}$ and $M$ but more than $1 / 2$ that between $R_{4+5}$ and $\mathrm{M}$; the robust epandrium that is higher than wide; the gonite that is retracted and invisible from a posterior view and that is separated beneath the aedeagus, and the thick phallapodeme. In Psilephydra, the facial setae are reduced, hair-like, the distance between $\mathrm{R}_{2+3}$ and $\mathrm{R}_{4+5}$ is equal to that between $\mathrm{R}_{4+5}$ and $\mathrm{M}$, the epandrium is as long as wide and the gonite is visible from a posterior view, the gonites loop beneath the aedeagus, and the phallapodeme is slender (Mathis 1982, Mathis and Zatwarnicki 1988). 
1. Face distinctly bicolored and protrudent on ventral $1 / 2$, dorsal surface of protrusion with some metallic blue coloration, ventral portion along oral margin densely microtomentose, whitish gray (Nepal) S. kaskiensis (Mathis)

- Face either unicolorous or with coloration changes gradual, anterior surface of ventral $1 / 2$ nearly flat, not conspicuously protrudent

2. Dorsocentral setae 4, anterior seta presutural; overall length 2.9-3.4 mm (Nepal) .........

- Dorsocentral setae 3, all postsutural; overall length 2.0-2.6 mm 3

3. Apex of gonite dentate (China: Sichuan) S. sichuanensis sp. nov.

- Apex of gonite smooth, not dentate (Japan and Ryukyu Islands)... S. fluvialis (Miyagi)

\section{Sinops sichuanensis sp. nov. (Figs. 1-15)}

Diagnosis. Two pairs of strong lateroclinate orb; 3-4 long facial setae. Three pairs of posts dc, without pres dc; acr weak and short, continuing in a posts row to base of scutellum; 1 strong presutural seta; posterior npl much farther from notopleural suture than anterior npl; 1 strong sa, psa absent; two pairs of sc. Forefemur with row of pv, mid femur with row of av. Male genitalia: Epandrium base as wide as apex in posterior view; cerci long, thickened at base and slender at apex. Gonite falcate at base in lateral view. Female genitalia: Cerci flatiron-shaped in lateral view, yellow at base and yellow at apex. Sternite 8 divided into 2 triangular sclerites.

Description. Male body length $2.2-2.4 \mathrm{~mm}$, wing length $2.5-2.7 \mathrm{~mm}$.

Head (Figs. 1, 3). Generally black, subshiny; anterior frons with transverse dent. Setulae and setae of head black. 1 lateral vertical seta as long as 1 medial vertical seta; 1 pair of strong ocellar setae; 4 lateroclinate orb, $1^{\text {st }}$ and $3^{\text {rd }}$ setae thin and short. Slightly projecting face black with greenish blue dense microtomentum, with 3 long facial setae as strong as oral setae on each side. Gena with 1 very strong seta. Antenna black; arista short pubescent.

Thorax (Figs. 2, 4). Subshiny black; mesonotum with thin gray microtomentum. Thoracic setulae and setae black. Three pairs of postsutural dc, posteriormost dc just lateral to dorsocentral line and just anterior to scutellum; pres dc absent; acr weak and short, continuing in a posts row to base of scutellum; 1 strong presutural seta; $2 \mathrm{npl}$, posterior $\mathrm{npl}$ much farther from notopleural suture than anterior npl; katepisternal seta weaker than anepisternal seta; 1 strong sa, psa absent; scutellum with two pairs of sc, apical sc about two times as long as lateral sc; anepisternum with 1 long seta; katepisternum with 1 strong seta on dorsal portion. Legs black, subshiny with gray microtomentum; tarsi brown. Setulae and setae on legs black. Forefemur (Fig. 6) with row of pv shorter than width of forefemur, midfemur (Fig. 7) with row of av. Pulvilli well developed; tarsal claws short, and distinctly 

yellowish.
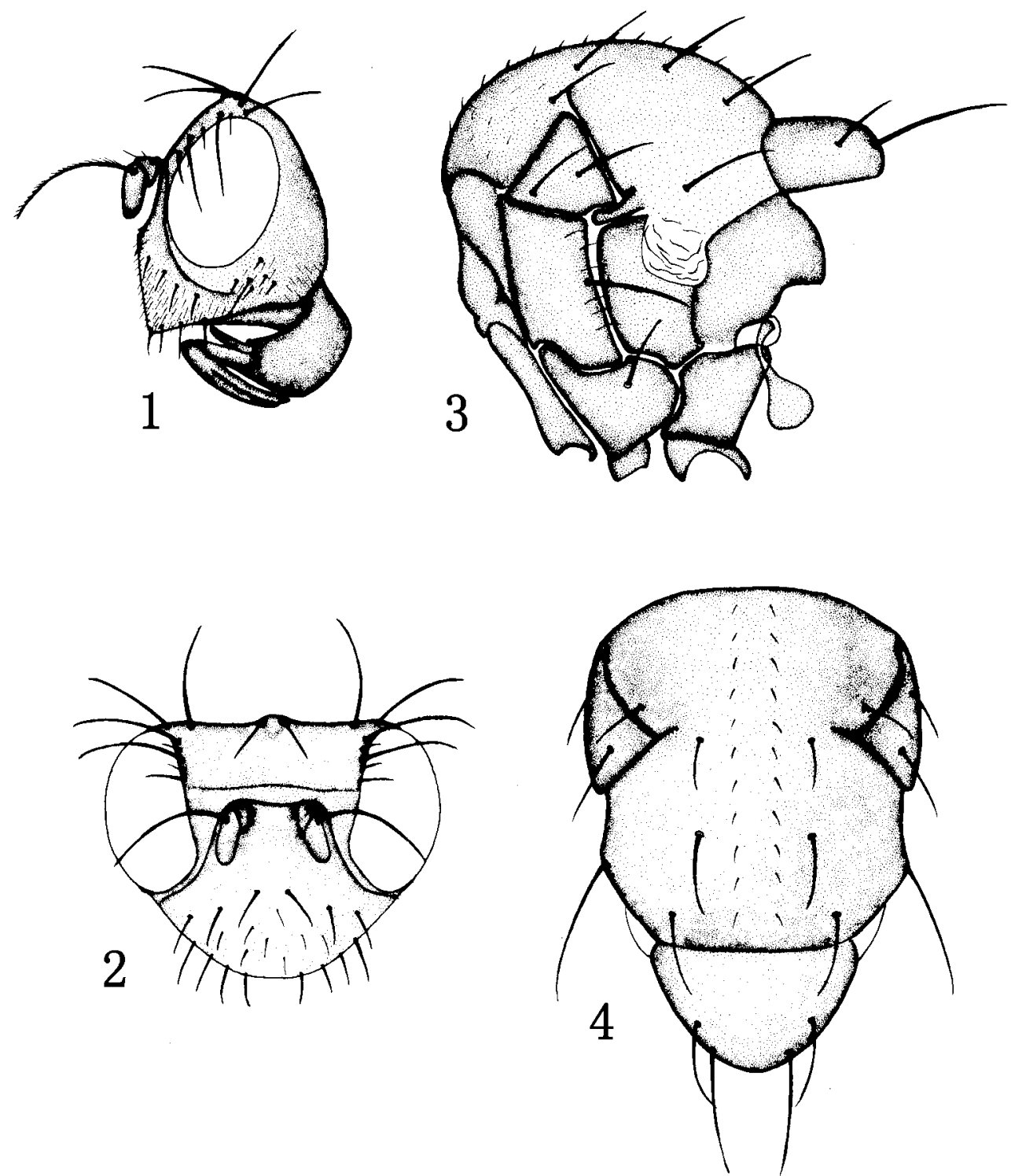

FIGURES 1-4. Sinops sichuanensis sp. nov. (male). 1. Head in lateral view; 2. head in anterior view; 3. thorax in lateral view; 4. thorax in dorsal view.

Abdomen subshiny black. Abdominal setulae and setae black.

Male genitalia (Figs. 9-12). Epandrium large and broad, parallel-sided in posterior view; cerci long, thickened at base and slender at apex; anterior gonite falcate at apex, posterior gonite with dentate margin at apex in lateral view. 
Female. Body length 2.6-2.7 mm, wing length 2.7-2.8 mm. Female genitalia (Figs.

13-15). Cerci subtrapezoidal in lateral view, brownish yellow at base and yellow at apex.

Sternite 8 divided into 2 triangular sclerites.

Specimens examined. Holotype ${ }^{\star}$, Sichuan: Emeishan Mountain, 16. VIII. 1998, leg. Ding Yang (CAU). Paratypes $30^{x} 0^{x}, 3$ 우우, same data as holotype (CAU \& USNM).

Distribution. China (Sichuan).

Etymology. The specific name refers to Sichuan, a province in south-central China.
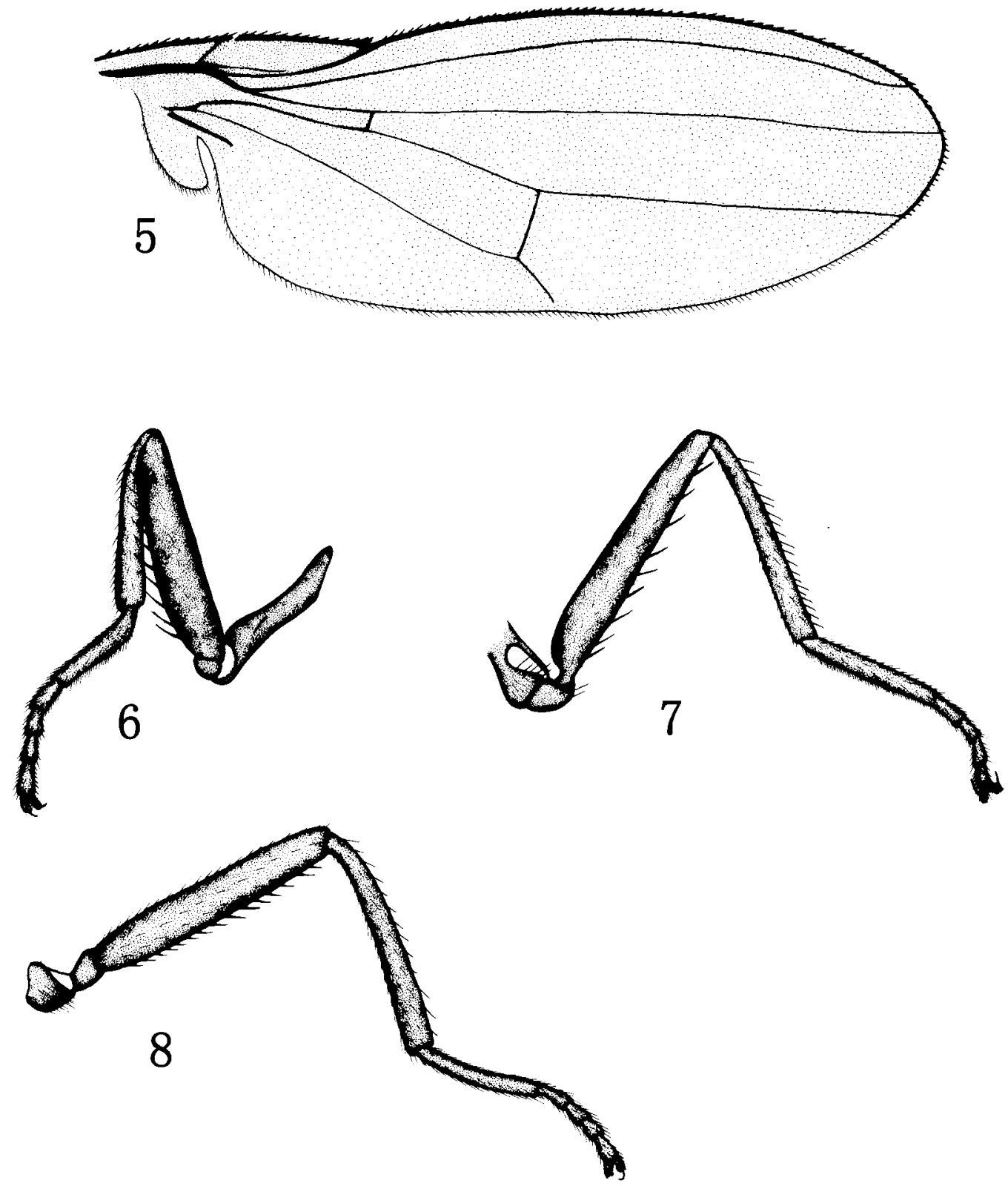

FIGURES 5-8. Sinops sichuanensis sp. nov. (male). 5. Wing; 6. foreleg; 7. midleg; 8. hindleg. 

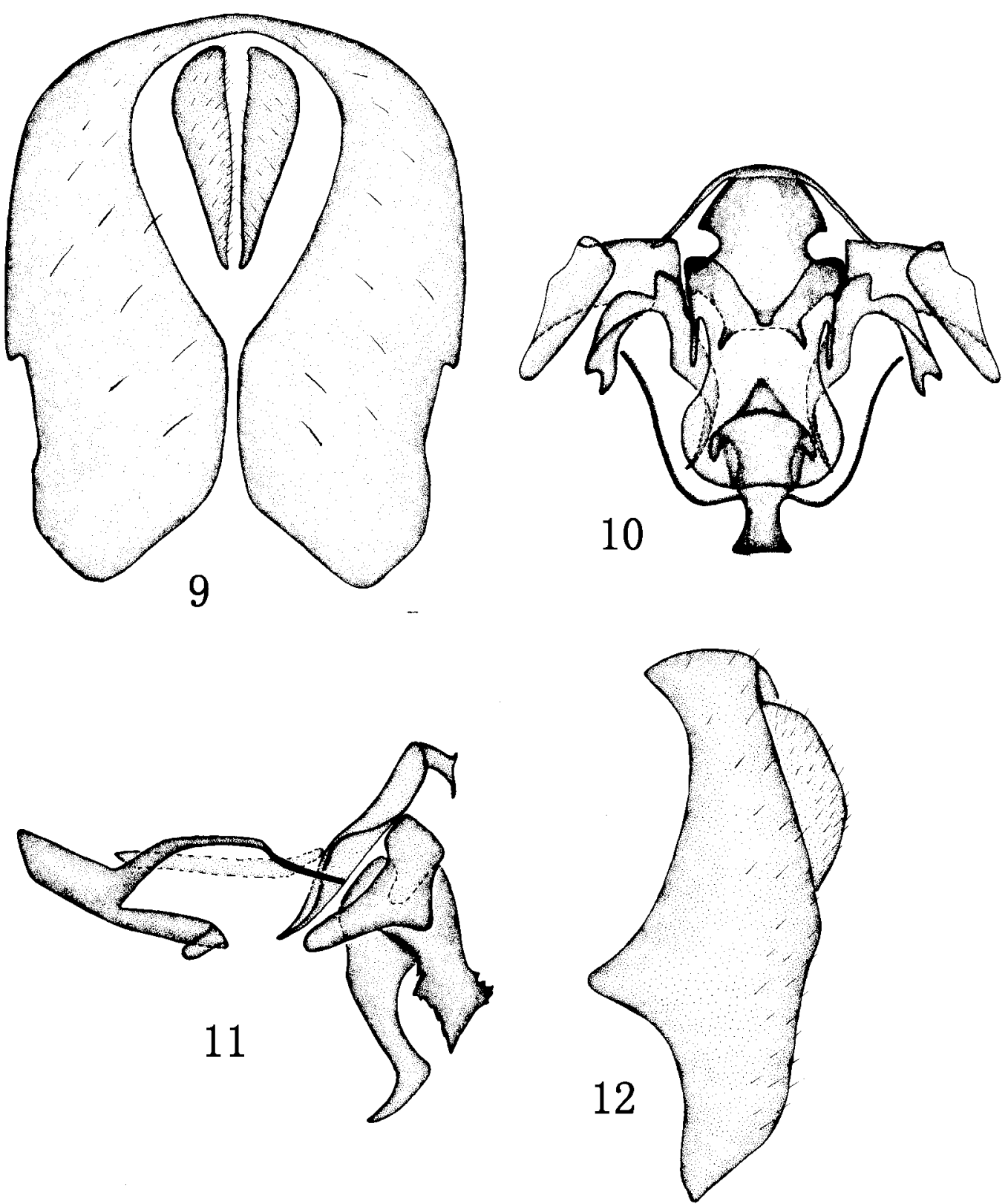

FIGURES 9-12. Sinops sichuanensis sp. nov. (male). 9. Epandrium in posterior view; 10. genitalia in internal view; 11. genitalia in lateral view; 12. epandrium in lateral view. 


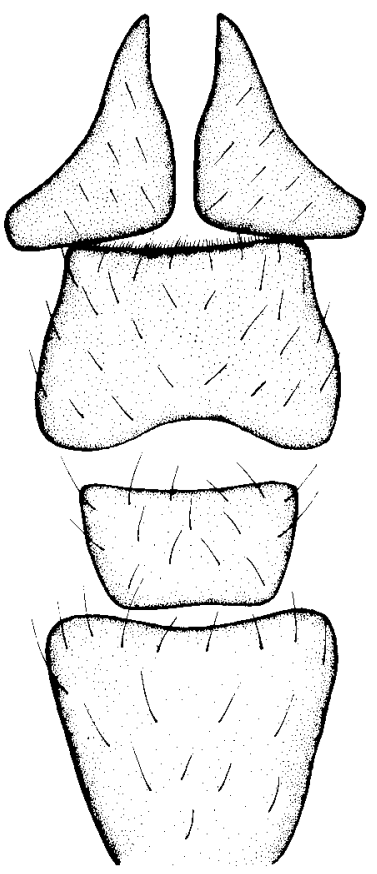

13

FIGURES 13-15. Sinops sichuanensis sp. nov. (female). 13. 5-8 sternites; 14. cerci in lateral view; 15. cerci in posterior view.

\section{Cladistic Analysis}

In the presentation on generic-level relationships that follows, the characters used in the analysis are noted first. Each character is immediately followed by a discussion to explain its states and to provide perspective and any qualifying comments about that character. After presentation of the information on character evidence, an hypothesis of the cladistic relationships is presented and briefly discussed. The cladogram (Fig. 16) is the primary mode to convey relationships, and the discussion is to supplement the cladogram and is intended only to complement the latter. In the discussion of character data, a " 0 " indicates the state of the outgroup; a "1" or " 2 " indicates the derived states. Characters 1, 2, 15, 16, $17,18,21,22$ and 23, which are autapomorphies for various genera, were made inactive (]) for the analysis so that they do not figure into the calculation of the consistency and retention indices. Characters 6,10 , and 14 are multistate characters and were made nonadditive (-) in the analysis. The numbers used for characters in the presentation are the same as those on the cladogram, and the sequence is the same as noted in the character matrix (Table 1). Five genera including Sinops from the tribe Dagini were selected as ingroup 
members; Coenia Robineau-Desviody and Scatella Robineau-Desviody were selected as outgroups. Twenty-four adult characters from the head, thorax and male terminalia were used in the cladistic analysis. Selected characters of four known genera for cladistic analysis are based on the descriptions made by Mathis $(1979,1982)$, Mathis and Zatwarnicki (1988) and Olafsson (1991).

\section{Characters Used in the Cladistic Analysis}

1) Facial vestiture: mostly bare (0); midface setulose (1)(this is an autapomorphy for the subfamily Ephydrinae). $0 \rightarrow 1$

2) Size of katepisternal seta: well developed (0); weaker than anepisternal seta (1)(this is an autapomorphy for the tribe Dagini). $0 \rightarrow 1$

3) Oral setae: without long setae (0); with long setae (1). $0 \rightarrow 1$

4) Facial setae: without strong setae on each side (0); with well-developed setae on each side (1). $0 \rightarrow 1$

5) Number of dc: 1-3 paired dc (0) (4 in S. nepalensis; apparently by convergence); 4 paired dc (1). $0 \rightarrow 1$

6) Arrangement of dc: $(1+2)(0) ;(1+3),(1) ;(0+3),(2)$ (this is a multistate character that was treated as nonadditive). $1 \rightarrow 0 \rightarrow 2$

7) Length of claw: short and curved (0); long and straight (1). $0 \rightarrow 1$

8) Pulvilli: present (0); reduced or lacking (1). $0 \rightarrow 1$

9) Vestiture of postpronotum: with macrosetae (0); lacking macrosetae (1). $0 \rightarrow 1$

10) Number of orb: 2 pairs (0); 1 pairs (1); 3 pairs (2) (this is a multistate character that was treated as nonadditive). $1 \rightarrow 0 \rightarrow 2$

11) Genal seta: distinct (0); reduce or lacking (1). $0 \rightarrow 1$

12) Position of posterior npl: at the same level as anterior npl (0); raised above level of anterior npl (1). $0 \rightarrow 1$

13) Ocellar seta: present (0); absent (1). $0 \rightarrow 1$

14) Apical position of vein $R_{2+3}$ : distance between $R_{2+3}$ and $R_{4+5}$ equal to that between veins $\mathrm{R}_{4+5}$ and $\mathrm{M}(0)$; distance between $\mathrm{R}_{2+3}$ and $\mathrm{R}_{4+5}$ less than $1 / 2$ that between veins $\mathrm{R}_{4+5}$ and $M$ (1); distance between $R_{2+3}$ and $R_{4+5}$ more than $1 / 2$ that between veins $R_{4+5}$ and $M$ (2) (this is a multistate character that was treated as nonadditive). $1 \rightarrow 0 \rightarrow 2$

15) Npl: anterior $\mathrm{npl}$ as long as posterior $\mathrm{npl}(0)$; anterior $\mathrm{npl}$ shorter than posterior $\mathrm{npl}$ (1)(this is an autapomorphy for the genus Psilephydra). $0 \rightarrow 1$

16) Forefemoral vestiture: without spine-like setae (0); with spine-like setae (1)(this is an autapomorphy for all taxa in the analysis). $0 \rightarrow 1$

17) Shape of epandrium in posterior view: longer than wide (0); as long as wide (1) (this is an autapomorphy for the genus Psilephydra). $0 \rightarrow 1$

18) Gonite in posterior view: invisible (0); visible (1) (this is an autapomorphy for the genus Psilephydra). $0 \rightarrow 1$ 
19) Development of phallapodeme: slender (0); thickened, plate-shaped (1). $0 \rightarrow 1$

20) Shape of face: distinctly protrudent (0); slightly protrudent (1). $0 \rightarrow 1$

21) Acr: with distinct presutural acr (0); distinct presutural acr absent (1)(this is an autapomorphy for the genus Scatella). $0 \rightarrow 1$

22) Size of posterior alar: smaller than anterior setae (0); reduced (1) (this is an autapomorphy for all taxa in the analysis except for Coenia). $0 \rightarrow 1$

23) Size of facial setae: hair-like (0); bristle-like (1) (this is an autapomorphy for the genus Psilephydra). $0 \rightarrow 1$

24) Gonite: looping below aedeagus (0); separated below aedeagus (1). $0 \rightarrow 1$

TABLE 1. Characters state distribution of characters used in cladistic analysis of Dagini.

\begin{tabular}{|c|c|c|c|c|c|c|c|c|c|c|c|c|c|c|c|c|c|c|c|c|c|c|c|c|}
\hline & & & & & & & & & & 1 & 1 & 1 & 1 & 1 & 1 & 1 & 1 & 1 & 1 & 2 & 2 & 2 & 2 & $?$ \\
\hline & 1 & 2 & 3 & 4 & 5 & 6 & 7 & 8 & 9 & 0 & 1 & 2 & 3 & & 5 & 6 & 7 & 8 & 9 & 0 & 1 & 2 & 3 & 4 \\
\hline Coenia & 1 & 0 & 1 & 0 & 1 & 1 & 0 & 0 & 1 & 0 & 0 & 0 & 0 & 0 & 0 & 1 & 0 & 0 & 0 & 0 & 0 & 0 & 1 & \\
\hline Scatella & 1 & 0 & 1 & 1 & 0 & 0 & 0 & 0 & 1 & 0 & 0 & 0 & 0 & 0 & 0 & 1 & 0 & 0 & 0 & 0 & 1 & 1 & 1 & \\
\hline Diedrops & 1 & 1 & 1 & 0 & 1 & 1 & 0 & 0 & 1 & 0 & 0 & 0 & 1 & 1 & 0 & 1 & 0 & 0 & 1 & 1 & 0 & 1 & 1 & 0 \\
\hline Psilephydra & 1 & 1 & 0 & 0 & 0 & 2 & 0 & 0 & 1 & 1 & 1 & 0 & 0 & 0 & 1 & $?$ & 1 & 1 & 0 & 1 & 0 & 1 & 0 & 0 \\
\hline Dagus & 1 & 1 & 1 & 1 & 1 & 1 & 1 & 1 & 1 & 2 & 0 & 1 & 0 & 0 & 0 & 1 & 0 & 0 & 1 & 1 & 0 & 1 & 1 & ? \\
\hline Physemops & 1 & 1 & 1 & 0 & 0 & $?$ & 0 & 0 & 1 & 1 & 1 & 1 & 0 & 0 & 0 & 1 & 0 & 0 & 1 & 1 & 0 & 1 & 1 & ? \\
\hline Sinops & 1 & 1 & 1 & 1 & 0 & 2 & 0 & 0 & 1 & 0 & 0 & 1 & 0 & 2 & 0 & 1 & 0 & 0 & 1 & 1 & 0 & 1 & 1 & 1 \\
\hline
\end{tabular}

\section{Results and Discussion (Fig. 16)}

Using the implicit enumeration (ie*) option of Hennig86, which is an exhaustive search, a single most parsimonious tree was generated from the analysis of the 24 characters. The cladogram has a length of 23 steps and the initial consistency and retention indices were 0.78 and 0.61 respectively. After successive weighting, the consistency and retention indices increased to 0.96 and 0.90 .

As indicated in the cladogram (Fig. 16), the tribe Dagini is a monophyletic lineage, and the new genus, Sinops, is nested within Dagini. The monophyly of the tribe Dagini is supported by a weaker katepisternal seta (character 2), thickened phallapodeme (character 19) and the more slightly protrudent face (character 20). The typology of the cladogram for lineages within Dagini is a simple sequence of nested lineages with the genus Diedrops forming the basal most, monophyletic clade. The monophyly of Diedrops is supported by the elevated insertion of the posterior npl seta (character 12) and the lack of an ocellar seta (character 13). Dagus is the next nested lineage and it is supported mainly by the length of the claws (character 7), the reduced or absent pulvilli (character 8) and having three orb (character 10). The monophyly of the new genus Sinops is supported by the apical position 
of vein $\mathrm{R}_{2+3}$ (character 14) and the shape of the gonite (character 24). The monophyly of the genus Psilephydra is supported mainly by the long oral setae (character 3), the shorter anterior npl seta (character 15), the forefemoral spine-like setae (character 16), the wide epandrium (character 17), visible gonite (character 18) and bristlelike facial setae (character 23). The status of Physemops, as a monophyletic clade, remains unsubstantiated.

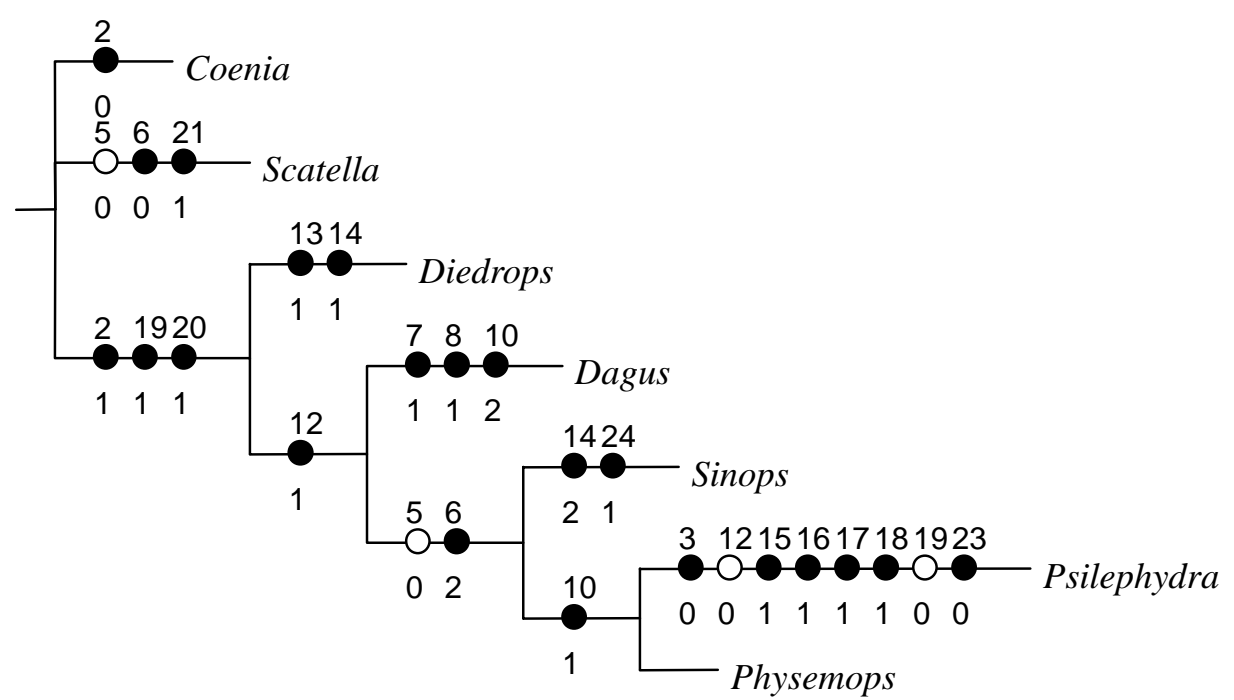

FIGURE 16. Cladogram depicting the hypothetical cladistic relationships among genera of the tribe Dagini. Filled squares represent unambiguous synapomorphies; open squares indicate ambiguous synapomorphies and parallelisms.

\section{Acknowledgments}

We are very grateful to Mrs. Lili Zhang, Mr. Xingyue Liu and Mrs. Mengqing Wang (Beijing) for their kind help in many ways. For reviewing an earlier draft of this manuscript, we thank Drs. James F. Edmiston and Tadeusz Zatwarnicki. This research is supported by the National Natural Science Foundation of China (No. 30225009).

\section{References}

Mathis, W. N. (1979) Ephydrinae (Diptera: Ephydridae): A new perspective. In: Deonier, D.L. (E d.), First Symposium on the Systematics and Ecology of Ephydridae (Diptera). The North American Benthological Society, Oxford, Ohio, pp. 47-60.

Mathis, W. N. (1982) Studies of Ephydrinae (Diptera: Ephydridae), VI: Review of the tribe Digini. Smithsonian Contributions to Zoology, 345, 1-30.

Mathis, W. N. (1986) Studies of Psilopinae (Diptera: Ephydridae), I: A revision of the shore fly genus Placopsidella Kertész. Smithsonian Contributions to Zoology, 430, iv+30 pp. 
Mathis, W. N. \& C. L. Hogue. (1986) Description of a new species of the shore fly genus Diedrops (Diptera: Ephydridae) from Colombia. Contributions in Science, Natural History Museum of Los Angeles County, 377, 21-26.

Mathis, W. N. \& T. Zatwarnicki. (1988) Studies on the systematics of the shore-fly tribe Dagini (Diptera: Ephydridae). Proceeding Entomological Society Washington, 90 (1), 106-121.

Mathis, W. N. \& T. Zatwarnicki. (1990a) A revision of the western Palearctic species of Athyroglossa (Diptera: Ephydridae). Transactions of the American Entomological Society, 116(1), 103-133.

Mathis, W. N. \& T. Zatwarnicki. (1990b) Taxonomic notes on Ephydridae (Diptera). Proceedings of the Biological Society of Washington, 103(4), 891-906.

Mathis, W. N. \& T. Zatwarnicki. 1995. World catalog of shore flies (Diptera: Ephydridae). Memoirs on Entomology, International. Associated Publishers, 4, 1-423.

McAlpine, J. F. (1981) Morphology and terminology adults. pp. 9-63. In: McAlpine, J. F., Peterson, B.V., Shewell, G. E., Teskey, H. J., Vockeroth, J. R., \& Wood, D. M. (coordinators): Manual of Nearctic Diptera, Volume 1, Research Branch, Agriculture Canada, Ottawa, Monograph $27,674 \mathrm{pp}$.

Miyagi, I. (1977) Ephydridae (Insecta: Diptera). In Fauna Japonica, 113 pp.

Olafsson, E. (1991) Taxonomic revision of western Palaearctic species of the genera Scatella R.-D. and Lamproscatella Hendel, and studies on their phylogenetic positions within the subfamily E phydrinae (Diptera, Ephydridae). Entomologica Scandinavica Supplement, 37, 1-100. 